Revista Jurídica del Departamento de Derecho Academia de Derecho Administrativo

Tercera Época Año2. No. 3 / Julio-Diciembre 2010

\title{
II. Equidad de Género en las Instituciones Educativas
}

\author{
Dra. Adria Velia GONZÁLEZ BELTRONES \\ Universidad de Sonora \\ adriag@sociales.uson.mx \\ XX Seminario Nacional: "Educación, Empoderamiento y Desarrollo" \\ Saltillo, Coahuila. Noviembre 2009. Mesa Educación
}

\begin{abstract}
Cada vez queda más claro que las instituciones del pasado son obsoletas y que nuevas formas de "governance" y de liderazgo han de ser aprendidas. ...Lo único constante será el aprendizaje y el cambio permanentes. (E.H Schein, "Leadership and Organizational Culture", en El Líder del Futuro)
\end{abstract}

Hablar de equidad de género implica recordar que desde sus inicios la historia de la humanidad ha estado sellada profundamente por el patriarcado. Lo cual ha generado una estructura social y cultural asentada en el predominio permanente de una parte de la población sobre otra por razón de sexo. Esto a su vez ha generado que a las personas y su función en la sociedad se les encasille en razón de su género. Es decir, se ha efectuado la división del trabajo, de espacios y de determinadas expectativas sobre habilidades, capacidades, destrezas, sensibilidades entre otros aspectos, de acuerdo con el género al que se pertenece. $Y$ en consecuencia, estos estereotipos tan arraigados resulta difícil anularlos de la percepción de las personas y de la realidad que nos rodea. Es decir, al día de hoy se ha arraigado imágenes distorsionadas de la mujer de manera tal que siguen marcando roles establecidos que priorizan su responsabilidad en el ámbito doméstico frente al profesional.

Ahora bien, de acuerdo a los principios de administración, en toda organización se requiere coordinar los diversos componentes que en ella participan para alcanzar los objetivos, Por lo que la función de coordinación surge como un elemento esencial en las instituciones. Si miramos a nuestro entorno y preguntamos: ¿a quién o a quiénes les corresponde asumir ese papel de coordinación? La respuesta que se ofrece como obvia no irá en dirección a las mujeres. Sin embargo, una de las condiciones esenciales de la práctica de la igualdad en el seno de las organizaciones, es el acceso de la mujer a puestos de dirección ${ }^{1}$ y si esto no es práctica común en las organizaciones educativas, el modelo de educación que se transmitirá, contribuirá a desarrollar una educación contraria a los planteamientos de la igualdad de oportunidades entre mujeres y hombres. $Y$ esto es importante porque lo que las futuras generaciones de hombres y mujeres aprendan en las instituciones educativas en las que pasan los primeros quince años de su vida, determinará su posición y actitud al efecto.

En este tenor, si la educación es una profesión en donde predomina el número de mujeres. ¿Por qué, sin embargo, las estadísticas persisten en demostrar que el número de mujeres en puestos directivos en instituciones educativas es mucho menor de lo que correspondería en proporción a su presencia en esta profesión?

\footnotetext{
${ }^{1}$ La Equidad de Género en las Cumbres y Conferencias de las Naciones Unidas subrayan tal aspecto como indispensable para avanzar en el respeto a los derechos fundamentales.
} 
Revista Jurídica del Departamento de Derecho Academia de Derecho Administrativo

Tercera Época Año2. No. 3 / Julio-Diciembre 2010.

Si bien son escasos los estudios sobre el acceso de las mujeres a puestos de dirección en las organizaciones educativas, lo que dificulta precisar si existe un modelo de liderazgo femenino, es decir, si las mujeres tienen un peculiar estilo de desempeñar el poder, de ejercer influencia en las organizaciones De estas investigaciones se puede extraer algunas conclusiones de los expertos sobre: liderazgo y dirección de las mujeres:

1. Las mujeres tienden a ejercer el liderazgo de una forma diferente a la mayoría de los hombres ${ }^{2}$ basado en su manera de pensar, sentir y actuar

2. la mujer recurre a consensuar y a mediar a la hora de ejercer el liderazgo.

Sin embargo, si bien afirman el rasgo señalado en el punto dos precitado, como algo positivo, no dejan de "aclarar" que también esto puede deberse a la "falta de seguridad personal e independencia de las mujeres". En ningún caso, esta "aclaración o particularidad", se da al analizar el liderazgo masculino, al que tradicionalmente se le atribuyen cualidades entre las que no están la "falta de seguridad e independencia". Pero tratándose de mujeres parece necesario minimizar incluso las apreciaciones positivas. Tradicionalmente se consideró que las mujeres no debían ejercer el liderazgo en las organizaciones porque se creía que poseían una serie de rasgos que resultaban contraproducentes para el ejercicio de esas funciones y que según Helgsen (1995) se resumen en los siguientes:

1) Demasiado centradas en dar importancia a los vínculos afectivos

2) Dificultad para considerar el mundo de la dirección como un "gran juego"

3) Escasa capacidad para captar lo esencial del trabajo en equipo, dado su poca afición a los deportes competitivos de equipos como el fútbol

4) Deberían desarrollar un mayor respeto hacia las estructuras jerárquicas y disminuir sus dudas sobre su efectividad

Sí se tuviera que considerar tales características como "rasgos" distintivos del liderazgo femenino en general, habría que cuestionar ¿si estas características no son las adecuadas para liderar y dirigir las organizaciones actuales?

Al respecto Al Khalifa (1999) afirma que la teoría "gerencial" aplicada a los centros educativos ha incrementado la posibilidad de asociar conceptos como liderazgo administrativo y masculinidad y, por supuesto, liderazgo y jerarquía. El modelo de "management" que se introdujo en los centros escolares procedente del mundo de la empresa en el Reino Unido, redujo notoriamente el número de mujeres que ocupaban cargos directivos en los centros educativos. $Y$ este es el modelo por el que, desgraciadamente, han apostado la mayoría de las administraciones educativas. Aunque Kaufmann $(1996,183)$ consideró en su momento que "en la actualidad, las mujeres están en condiciones de revolucionar el lugar de trabajo, precisamente no por dejar a un lado sus valores tradicionales, sino precisamente por expresarlos, en su cotidianeidad laboral", al parecer en matera de educación no han llegado los mejores tiempos para el liderazgo femenino.

Los rasgos más destacados según los expertos y que hacen diferente el uso del poder por parte de las mujeres serían los siguientes:

\footnotetext{
${ }^{2}$ (Apellániz, 1997; Coronel y otros, 1999; Santos Guerra, 2000, etc.),
} 
Revista Jurídica del Departamento de Derecho Academia de Derecho Administrtativo

Tercera Época Año2. No. 3 / Julio-Diciembre 2010.

1. Un estilo más democrático, dialogante, pedagógico, consensuador y mediador. Una actitud más receptiva y participativa.

2. Un liderazgo más multidireccional y multidimensional, favoreciendo el desarrollo de valores y acciones colectivas.

3. Un ejercicio del liderazgo más firme y constante.

4. Más creativas en las propuestas para ejercer la dirección.

5. Más comunicativas y abiertas a las relaciones personales, a las propuestas de sus compañeras y compañeros, a escuchar sin enjuiciar de una forma más comprensiva.

6. Desarrollan políticas de cooperación y participación.

7. Potencian relaciones interpersonales.

8. Disponibilidad para el cambio.

9. Crean un clima de colegialidad y gestión colaborativa con el profesorado.

10. Construyen redes informales, propiciando la cohesión y los vínculos entre los miembros, alentando a otros a compartir los recursos.

11. Les preocupa el abuso del poder y utilizan la coacción como último recurso.

12. Potencian el "aprender con los demás".

13. Clara preferencia por enfoques consultivos y cooperativos.

14. Habilidad para combinar y reconciliar expectativas y experiencias contradictorias, promoviendo el compromiso colectivo de los miembros de la organización.

15. Desarrollo de políticas de cuidado y apoyo mutuo.

16. Mayor atención a los sentimientos y al uso de una "inteligencia emocional" más sensible a las emociones y situaciones personales de los demás.

En síntesis, dice Kaufmann (1996), las mujeres ponen la parte emocional también en el trabajo. En general las mujeres se hallan más capacitadas para tener en cuenta el lado "humano" de las personas desarrollando para ellos sus dotes intuitivas naturales.

Si son estas las características que tienden a definir el ejercicio de las mujeres cuando tienen poder, tendríamos entonces que preguntarnos qué pasaría si los cargos de influencia y poder en el mundo estuvieran en manos de las mujeres. Algunas mujeres y sus adeptos, afirman que la revolución pendiente es la "revolución de las mujeres". Y que si los valores asociados tradicionalmente a las mujeres tuvieran mayor reconocimiento social dejarían de existir guerras, industria bélica y la cultura machista que hoy por hoy domina y conforma el ejercicio del poder.Frente a esta argumentación, algunos autores afirman que los ejemplos de mujeres ejerciendo cargos de poder o de influencia en la sociedad contemporánea no han sido precisamente ejemplos de este tipo de ejercicio del poder, sino que han sido más duras y más implacables que los hombres. Y ahí está el ejemplo de Margaret Thatcher en Inglaterra, Condoleezza Rice en USA por citar las más relevantes en nuestros días al respecto.

En abono de lo anterior habría que recordar que en la sociedad actual, tal como está organizada, las pocas mujeres que acceden al poder lo tienen que hacer demostrando que son más "duras" que los propios hombres. Para llegar a esos cargos de influencia han tenido que socializarse y mimetizarse en una cultura machista, empleando los mismos métodos y aún más duros que los que han empleado sus competidores del sexo opuesto No hubieran sido admitidas en caso contrario.

"La mujer muchas veces asume modelos o patrones masculinos que la sociedad le ha hecho ver como los adecuados para conseguir el éxito. Una mujer firme, franca y directa se la considera agresiva o con demasiadas aspiraciones, lo cual no se aplica a los hombres que muestran el mismo comportamiento. Por todo ello vemos que las mujeres, cuando ejercen cargos de responsabilidad, tienen no sólo que distanciarse de las otras mujeres, sino que tienen que aislar su yo emocional de los hombres" (Coronel Llamas, 1996). 
Revista Jurídica del Departamento de Derecho Academia de Derecho Administrativo

Tercera Época Año2. No. 3 / Julio-Diciembre 2010

De otra parte, cabe señalar que cuando una mujer tiene una posición de poder, las críticas que sufren no suelen ser argumentadas, sino que son utilizadas para descalificarlas dirigiéndose más al yo emocional que al racional ("está histérica", "es que tiene problemas en su casa", por citar algunas expresiones.). Son críticas dirigidas más a la persona, que a su labor de gestión. Esto se puede ver también cuando en una organización dos mujeres, con cargos de poder, compiten por el liderazgo. Se tiende a interpretar como "cuestión de celos" Mientras que en el caso de los hombres se considera "lucha" por el poder.

No obstante lo anterior, desde la legislación y la contienda en distintos ámbitos de muchas mujeres se está abriendo una brecha de posible "normalidad futura" en la visión de las mujeres ejerciendo el poder en nuestra sociedad. $Y$ esto hará posible que esas mujeres cambien la forma y el estilo de ejercer el poder en las organizaciones de las que formen parte. Al respecto coincidimos con (Kaufmann, 1996, 186) en su aseveración simplificada según la cual "en las organizaciones modernas, y con entornos turbulentos y cambiantes, como los que predominan en la mayoría de las sociedades avanzadas, una estructura menos formal y más flexible resulta indispensable. Se sustituiría la ideología militar subyacente por una ideología 'ecológica', enfatizando la importancia de la interpelación entre las cosas y personas. Por todo ello podemos concluir que las mujeres pueden llegar a ser mejores directivas que los hombres por su experiencia de implicación activa en la esfera doméstica, que les proporciona una amplia experiencia en la gestión de conflictos, enseñar, guiar, impartir información, gestionar demandas contradictorias, entre otros".

Pero, para que las mujeres puedan ejercer este liderazgo alternativo, aún hay un obstáculo previo que solucionar: el acceso de las mismas a esos puestos de poder. Si como afirman los expertos, el liderazgo femenino cumple las expectativas de lo que debería ser un liderazgo positivo, tal, ¿por qué los cargos relevantes son desempeñados mayoritariamente por hombres? Tal como demuestran las estadísticas, en una sociedad, donde la igualdad de oportunidades entre mujeres y hombres parece alcanzada en la mayoría de las normas jurídicas fundamentales, en la realidad que se vive día con día en nuestras organizaciones, parece una contradicción que no haya paridad en el ejercicio de la dirección en todas las organizaciones e instituciones. Esto es especialmente significativo en las instituciones educativas, donde las mujeres son mayoría entre los profesionales que ejercen su trabajo en ellas y $\sin$ embargo, la proporción de mujeres en cargos directivos es minoritaria.

Así, previo a la posibilidad de este tipo de ejercicio de la dirección y el liderazgo de forma alternativa o diferente cabe preguntar: ¿por qué en cargos de responsabilidad y de dirección hay tan pocas mujeres? o ¿qué es lo que obstaculiza a las mujeres incursionar en el desempeño de puestos directivos $\mathrm{o}$ a liderar instituciones educativas?

Aportes de los especialistas

Al analizar los especialistas este aspecto en las instituciones educativas, por ser uno de los contextos organizativos más relevantes al respecto ${ }^{3}$, aunado a los datos que aportan las estadísticas oficiales se muestran persistentes año a año: que el número de mujeres en cargos directivos en las instituciones educativas es sensiblemente inferior al que le correspondería en proporción a su presencia en esta profesión.

\footnotetext{
${ }^{3}$ toda vez que la profesión educativa tiene un alto porcentaje de mujeres ejerciéndola respecto al número de hombres que trabajan en este campo, especialmente en las etapas inicial, preescolar y primaria dela educación básica.
} $\sim 9 \sim$ 
Revista Jurídica del Departamento de Derecho Academia de Derecho Administrtativo

Tercera Época Año2. No. 3 / Julio-Diciembre 2010

En Sonora, según se señala en el Informe General de Resultados educativos 1998-2003 de la Secretará de Educación y Cultura hay en el nivel de educación inicial y básica ${ }^{4}$, un total de 17.484 profesores y profesoras que ejercen la docencia en educación infantil, primaria y educación especial en toda el estado, de los que $\mathbf{5 8 . 6 \%}$ son mujeres y el $\mathbf{4 1 . 4} \%$ restante hombres. $Y$ aun cuando en dicho informe se señala que "para disipar dudas sobre avance magisterial convine informar que "la gran mayoría de los cargos directivos son ocupados por maestros en razón de los procesos escalafonarios que están establecidos, pero que por ello cambian de categoría de "docente" a "directivos"', en las cifras no se separa el número de puestos por género y aunque este porcentaje es diferente en función del nivel educativo, en las estadísticas nacionales se puede observar que : a mayor nivel educativo menor proporción de presencia femenina hay en puestos de dirección.

No sólo es más frecuente encontrar hombres en las tareas de dirección en los niveles superiores del sistema educativo estatal (secundaria frente a primaria o inicial), sino que también nos los encontramos con más frecuencia en los centros más grandes y con mayor número de alumnado y profesorado. Estos datos parecen ser similares en todo el país ${ }^{5}$.Parece pues que las mujeres ocupan más el puesto de dirección en las instituciones de educación primaria y preescolar de dimensiones más reducidas. Algunos autores (Coronel, Moreno y Padilla, 1999) lo explican por el descenso de prestigio del cargo de dirección en educación. Dado que los hombres tienden a competir por las profesiones más prestigiosas y lucrativas, cada vez van dejando más espacios "desechables" a las mujeres (en este caso, en centros educativos de primaria e infantil). No obstante, como estos mismos autores dejan notar citando a Prichard y Deem (1999), hay que ser críticos con el incremento de la participación de las mujeres en los puestos de gestión intermedios de la educación, en parte por considerar que las razones no son otras que su mejor adaptabilidad a los nuevos requerimientos que buscan una gestión más flexible y menos burocrática como requisito para la reestructuración de los servicios públicos. De ahí que la feminización no deba dejar de comprenderse como un efecto de esa presión que el nuevo modelo de gestión (descentralizado, competitivo y flexible) viene a imponer.

A pesar de estas verificaciones, lo cierto es que el número de mujeres en puestos de dirección en centros escolares es mucho menor que el que les correspondería en proporción a su presencia. La diversidad de respuestas que se han argumentado desde las investigaciones de los autores precitados se puede resumir en las siguientes fuentes:

1) Histórica, social y cultural. En relación a la menor proporción de mujeres que acceden a cargos directivos no tiene que ver únicamente con el mundo de la educación, sino que es una constante social en todos los campos profesionales actuales: la costumbre social que se convierte en norma a la que todos/as nos sentimos inclinados a amoldarnos para no destacar ni hacernos notar. Como se mencionó al principio de esta presentación, la función de la mujer ha estado reducida de forma tradicional al ámbito de lo domestico y privado, y por ello cuando accede al mundo laboral y al mundo público se encuentra con dificultades no sólo a nivel personal, sino por parte del entorno más próximo (familiar) y del entorno sociocultural más amplio (las organizaciones).

Según Nicolson (1977), para la mujer que logra el acceso a cargos de responsabilidad probablemente aumentarán los problemas y tensiones en su vida. Y no sólo debido a la presión que supondrán las opiniones de su entorno más próximo (pareja, familia...), sino también por la influencia de las redes de apoyo con las que puede contar tanto a nivel práctico como a nivel psicológico (otras mujeres, amistades, etc.). La concepción tradicional mayoritaria en estos dos ámbitos tenderá a orientarla hacia la reducción de sus ocupaciones al ámbito privado.

\footnotetext{
${ }^{4}$ La educación inicial y básica comprende 15 años, desde los primeros días después del nacimiento, hasta la educación secundaria para adolescentes.
} 
Revista Jurídica del Departamento de Derecho Academia de Derecho Administrativo

Tercera Época Año2. No. 3 / Julio-Diciembre 2010.

Asimismo, y de forma muy importante están las influencias ideológicas, que provocan consecuencias psicológicas en la mujer las mas de las veces de carácter negativo, en función de lo que la sociedad espera de ellas como madres de familia, esposas, hijas...., lo cual tiende a generar angustia, ansiedad por tener sentimientos de abandono hacia la educación de sus hijos/as; ya que el papel adjudicado a la mujer ha sido el de madre que no puede ser suplantado por otra figura de apego familiar.

Por otro lado, a nivel social, persiste una crítica bastante extendida, hacia las mujeres que anteponen el éxito profesional al papel preponderante que se le ha asignado histórica y socialmente: la maternidad. La sociedad plantea y define entonces, que masculinidad es equivalente a independencia y autonomía, en tanto que feminidad significa colaboración, actitud maternal y dependencia.

Un ejemplo de lo anteriormente señalado lo es que las mujeres en puestos de dirección no tienen hijos o es mínimo el número de hijos y máximo el espaciamiento entre ellos, comparado con el número de hijos y espaciamiento de los que procrean los hombres en esas mismas posiciones. Porque mientras que para la empresa un hombre con matrimonio y familia se considera como algo positivo que asegura la estabilidad, una mujer que tiene o puede tener hijos/as son una posible carga -permisos por embarazo, horas de dedicación al trabajo, prioridad en las decisiones, etc.-, lo cual acaba siendo asumido por las propias mujeres y vivido como "impedimento" en su carrera profesional.

2) Concepto de dirección y liderazgo tradicionalmente ligado a un enfoque jerárquico e individualista. En relación a esta segunda fuente, los expertos señalan que tiene como referencia y modelo en la mayoría de los casos la organización industrial. Es un estilo que evalúa el funcionamiento del centro sólo en relación con los resultados obtenidos. En el que la especialización y la división de funciones de los miembros. El/la directivo/a se convierte en un gestor de la organización. Este es un modelo esencialmente masculino. De hecho, los rasgos que se han venido definiendo como propios de este tipo de dirección y liderazgo siempre han estado asociados a las características que tradicionalmente se han atribuido a los hombres (determinación, fuerza, seguridad en sí mismo, etc.). Como dice Santos Guerra (2000) la función de la dirección en los centros educativos se ha identificado con la de quien tiene que garantizar el orden, y así la concepción de autoridad tiene que ver más con el poder que con la educación. "La visión de la dirección que se sustenta en la imposición, en la fuerza, en el dominio; esa cultura atribuye esas características a los varones".

El problema es que esto que, en principio, parecería algo que deberíamos desterrar de nuestras instituciones, tendiendo hacia los modelos de liderazgo compartido, sigue presente en el funcionamiento cotidiano de nuestros centros educativos. Lo que justificaría que el cargo de dirección en los centros educativos sigue siendo un cargo de poco interés para las mujeres en general y para aquellos hombres que se planteen un tipo de liderazgo compartido.

Si como señalan los expertos, el estilo de liderazgo femenino es un estilo colaborativo, abierto, democrático, consensuados, de trabajo en equipo y de compartir responsabilidades y trabajo, la mujer no se identifica, por tanto, con la palabra líder. La mujer no busca tanto el éxito social que parece proporcionar el poder o liderazgo. Pero sí sería más fácil que se planteara acceder a un cargo de dirección cuyo desempeño conllevara fundamentalmente una dinámica de diálogo, de negociación, de trabajo en equipo, de cuidado de las relaciones, de desarrollo la inteligencia emocional, etc. Por eso no es deseable para las mujeres acceder aun cargo que hoy por hoy lo que supone es asumir un rol de autoridad jerárquica (más en sintonía con el estilo masculino), que tiende a deteriorar el clima de entendimiento con sus compañeros y compañeras profesionales, y que muchas veces llega a crear distancia y enfrentamiento afectivo. 
Revista Jurídica del Departamento de Derecho Academia de Derecho Administrativo

Tercera Época Año2. No. 3 / Julio-Diciembre 2010

3) No querer acceder a puestos de dirección como un mérito personal en el currículum, como una conquista individual del poder se ha señalado como una tercera fuente de la menor proporción de mujeres en cargos directivos en el sector profesional de la educación. Es esta una de las diferencias básicas en el acceso de las mujeres a los puestos de dirección en las organizaciones educativas ya que tienden a planteárselo como un servicio a la comunidad educativa, como una aportación personal en el intento de mejorar la organización. Esta actitud se halla vinculada con el desarrollo de una intensa racionalidad basada en la competitividad, aprendida desde edades tempranas en los hombres. A los niños (varones) se les suele enseñar, durante su proceso de socialización que lo más importante es ganar, como valor primordial que se halla por encima de la valoración de los vínculos personales o el propio crecimiento. El énfasis está centrado en el "logro profesional", lo cual implica que el tiempo que comparten con sus familias es bastante limitado y sus casas se convierten en "sucursales" de sus despachos, en donde continúan el trabajo que no les ha dado tiempo a terminar en su despacho. Es decir, los hombres pretenden básicamente acceder a la cumbre de la organización, tener un cargo de poder y de representación institucional, a diferencia de las mujeres que en la concepción de su vida laboral, supone una visión de querer estar, no en la cumbre, sino en "el centro de la organización".

En diversas investigaciones realizadas se ha descubierto que son los hombres los que mayoritariamente presentan candidatura para ser directores en los centros. Son ellos los que muestran más interés por acceder a ese cargo. Parece pues claro que la lucha por el poder es más manifiesta y explícita en los hombres. Mayoritariamente, cuando la mujer se presenta al cargo de dirección no hay ningún otro candidato. Además, se ha comprobado que las mujeres dan menor importancia, a la hora de acceder a la función directiva, la remuneración que conlleva o la liberación de la docencia que lleva aparejada, mientras que toman más en cuenta la satisfacción personal y la experiencia adquirida que puede suponer la dirección.

4) Expectativas sobre la manera a ejercer el cargo de dirección de los hombres y las mujeres, dando por sentado que los hombres se ajustarán más al modelo de dirección que se espera de ellos. Se da por supuesto que las mujeres lo harán peor que los hombres, pues ellos "están más acostumbrados y entrenados tradicionalmente a ejercer el poder". Los resultados de investigaciones al respecto señalan que en igualdad de condiciones profesionales, la mujer es discriminada para acceder a un puesto de dirección porque "se presupone la valía superior del hombre".

Por una parte se considera que los hombres tienen ventajas para acceder y desempeñar un cargo directivo $y$, por otra, se afirma que las mujeres no tienen ninguna. Las tres ventajas que se citan textualmente de los hombres son: "mayor credibilidad por parte del profesorado y alumnado a los directores hombres"; "mayor respeto e incluso más miedo al enfrentamiento directo con los hombres, por lo que el profesorado se vuelve más cumplidor"; y "parece que es más capaz de imponer orden, a los ojos de los alumnos/as e incluso a veces de profesores/as". También se afirma que otros motivos de la discriminación por razón de género para acceder a un puesto de dirección por parte de las mujeres es que "hay una mayor confianza en la capacidad de toma de decisiones de los hombres", que "se considera que las mujeres tienen inseguridad y miedo para asumir responsabilidades" y "que se acepta más una orden o una corrección si viene de un director hombre"

"Cuando un hombre realiza mal la tarea de la dirección se suele argüir que es incompetente, que es poco responsable o que tiene problemas que le impiden hacer un buen trabajo. Cuando se trata de una directora, la causa de su mal planteamiento radica en su condición de mujer. (...) Se parte de una expectativa menor por parte de la familia y del entorno sobre la mujer, de una presión diferente sobre ella y de unas valoraciones distintas en el caso de que pretenda alcanzar algo tan legítimo y lógico para sus compañeros varones" (Santos Guerra, 2000, 62). 
Revista Jurídica del Departamento de Derecho

Academia de Derecho Administrativo

Tercera Época Año2. No. 3 / Julio-Diciembre 2010

Por lo que podemos constatar que se tienden a mantener los estereotipos y los prejuicios arraigados en el inconsciente colectivo sobre los comportamientos de ambos sexos en situaciones de poder: "se aplican a la mujer algunos estereotipos especialmente negativos para el ejercicio de la dirección. Las mujeres son consideradas histéricas, indiscretas, asustadizas, frágiles...” (Santos Guerra, 2000, 62). Se mantienen estereotipos sobre la mujer y sobre las mujeres: no pueden trabajar juntas, no confían unas en otras, se "despellejan", etc. Pero como dice Nicolson $(1997,196)$ "es la perspectiva masculina la que cree que las mujeres no pueden trabajar juntas". Esto hace referencia a la discriminación interna, la discriminación cultural, los límites que nos autoimponemos desde una tradición cultural que nos marca y nos limita.

5) Falta de modelos de identificación de mujeres en el poder es la quinta fuente que los expertos señalan como determinante para que lo ejerzan con características diferentes al de los hombres y que sean referentes para otras mujeres. Y, como plantea Santos Guerra, "Ios procesos de socialización constituyen un condicionante muy potente en una institución como la escolar, tan asentada en patrones de comportamiento culturales. La mujer ha estado tradicionalmente excluida de puestos de poder" (2000, 61) masculinos. Como se señaló, los "rasgos" y características que se asocian a los líderes, son más acordes con los que tradicionalmente se han atribuido a los hombres. Lo cual parece indicar que es importante el entrenamiento para poder desempeñar un puesto directivo. En la medida que se forma parte de un equipo de dirección y se van conociendo sus funciones y se va quitando el cierto recelo o miedo a su ejercicio, será más fácil volver a participar en un cargo directivo. Esto es importante para las mujeres, dado que si no se entrenan en la participación en estos cargos, se convierte en mucho más difícil el acceso a los mismos. Esta es una de las barreras que se constatan en diversos estudios. El entrenamiento en tomar decisiones, en organizar, hablar en público, el entrenamiento en decidir, etc., son aprendizajes fundamentales para ejercer los puestos directivos, en los que tradicionalmente se les ha formado mucho menos a las mujeres. Esta falta de modelos de identificación, se constata en el propio uso tradicional del lenguaje. De hecho el propio lenguaje niega esa posibilidad de identificación al no poder poner en femenino determinados cargos. Es sorprendente que en la profesión educativa, donde el modelo imperante debería ir por delante en cuanto a ruptura de diferencias o connotaciones discriminatorias por género, se siguen utilizando términos masculinos para designar cargos aún cuando estén desempeñados por mujeres: jefe de departamento, rector, etc"., esto es algo que viene pasando en la mayoría de las investigaciones y estudios genéricos (que no aborden específicamente el tema de género) sobre la dirección y el liderazgo.

"Las instituciones escolares, aunque mixtas, pueden calificarse de organizaciones androcéntricas. La definición de la escuela refleja los valores y significados de la cultura de los hombres. El lenguaje y la estructura de la educación son modelados, de forma predominante, por el patriarcado" (Santos Guerra, 2000, 56).

6) Una sexta fuente de la situación actual en cuanto a la proporción de mujeres que hay en puestos directivos en las organizaciones educativas hace referencia a la convicción de que actualmente ya" no hay discriminación" en la sociedad debido al doble discurso presente en el análisis de la realidad. Mientras el discurso dominante formal en las organizaciones está basado en la neutralidad e igualdad, la persistencia de asimetrías en relación al género indica la continuación o incluso el reforzamiento de la desigualdad de género en las organizaciones), en su funcionamiento cotidiano, en su cultura. Estamos en una cultura tradicionalmente tan patriarcal que no nos permite pensar una sociedad en condiciones de igualdad. No hay experiencias, ni referentes, ni tradiciones que nos muestren cómo sería una sociedad organizada paritariamente. $Y$ tendemos a negar las realidades que no se ajustan a las concepciones que consideramos que "deben ser". Y esto se perpetúa, incluso en los argumentos que se dan por parte de algunas mujeres y bastantes hombres que aluden a que ya hay mujeres en puestos directivos y que si no hay más será porque no quieren. Esto es lo que Santos Guerra (2000) denomina el “mito de la excepción” 
Revista Jurídica del Departamento de Derecho Academia de Derecho Administrativo

Tercera Época Año2. No. 3 / Julio-Diciembre 2010.

7) La séptima fuente que pretende explicar esta desigualdad por razón de género en el acceso de las mujeres a los puestos de dirección hace referencia a la baja autoestima que se ha fomentado tradicionalmente en las mujeres ante el ejercicio del poder. Esto tiene que ver con las percepciones en torno a las tareas directivas y cómo éstas afectan a la hora de decidir participar o no, "colaborar" o no. Y estas percepciones tienen mucho que ver con la idea de poder asociado a ellas y la crelación que tradicionalmente las mujeres han ido estableciendo con éste. Esto ha sido especialmente estudiado en las organizaciones empresariales donde la mujer consideraba que iba a ser menos respetada y valorada por sus subordinados que otros hombres en cargos directivos "en general, sólo se acepta y valora el modelo masculino en los puestos directivos, mientras que la forma habitual de las mujeres de ejercer el poder (consenso, diálogo, atención a diferentes puntos de vista) es visto como un indicativo de debilidad e inseguridad".

A manera de conclusiones quedan muchas preguntas sin respuesta inmediata:

Cabe preguntar de nuevo ¿ Si otra educación sería posible si las mujeres ejercieran el poder en las organizaciones educativas?.

¿Cómo es posible que haya tan pocas mujeres en puestos directivos por elección de sus compañeros y compañeras?.

¿O es que lo que en el fondo se demanda en los centros educativos es un director que mande, que imponga disciplina $y$ orden con autoridad personal que nos permita permanecer cómodamente apoltronados sin tener que arriesgarnos a implicarnos y tomar decisiones, criticando desde la retaguardia?

¿Cómo lograr que la mujer participe en el ámbito de la dirección educativa activa y críticamente sin tener que realizar un intenso esfuerzo para superar una serie de barreras sociales y culturales que son las que parecen impiden acceder y desarrollar un puesto directivo?.

Lo anterior supone, estudiar y comprender el papel de hombres y mujeres dentro de las organizaciones educativas. También es necesario, trabajar con mujeres para conocer qué hacen en el desempeño de cargos directivos, y para conocer su conquista, su adquisición y la generación de poder. No se debe permitir que en la teoría organizativa siga prevaleciendo ideología y valores masculinos, en función de los cuales los aspectos relacionados con el género femenino quedan relegados a un segundo plano.

Si se logra suprimir este tipo de barreras, lograremos que las organizaciones educativas del futuro tengan como eje principal a la persona y estén formadas por seres humanos integrales, siendo esto uno de los principales y más importantes retos tanto de mujeres como de hombres, y en general de nuestra sociedad.

Referencias Bibliográficas

AL KHALIFA, E. (1989). Management by Halves: Women Teachers and School Management. En Lyon, H. y Migniuolo, F.W. (Ed.). Women Teachers. Issues and Experiences. Milton Keynes: Open University Press.

ALVAREZ,M. Y SANTOS, M (1996). Dirección de centros docentes: gestión por proyectos, Madrid: Escuela Española.

ASTIN, H. y LELAND, C. (1991). Women of influence women of vision. San Francisco: Jossey-Bass. 
Revista Jurídica del Departamento de Derecho Academia de Derecho Administrativo

Tercera Época Año2. No. 3 / Julio-Diciembre 2010.

BALL, S.J. (1989). La micropolítica de la escuela. Hacia una teoría de la organización escolar. Madrid: Paidós-

MEC.

BASS, B.M. (2000). El futuro del liderazgo en las organizaciones que aprenden, En AA.VV. Liderazgo y organizaciones que aprenden (III Congreso Internacional sobre Dirección de Centros Educativos 331361) Bilbao: ICE Universidad de Deusto.

BENSCHOP, Y. y DOOREWAARD, H. (1998) Covered by equality: The gender subtext of organizations. Organization Studies, 19 (5) 787-805.

BOLÍVAR, A. (2001). Liderazgo educativo y reestructuración escolar. Ponencia en el I Congreso Nacional sobre Liderazgo en el Sistema Educativo Español (95-130). Córdoba: Grupo de Investigación sobre Liderazgo Educativo.

BRUNNER, C. (2000) Unsettled moments in settled discourse: women superintendents' experiences of inequality. Educational Administration Quarterly, 36 (1) 76-116.

BRUNNER, C. (1998) Sacred dreams: Women and the superintendecy. New York: State Univ. of NY Press.

CARRASCOSA, A. (2002). Leyes masculinas con voz femenina. Equis Equis, 3, 13.

CERRADA, A. y SEGURA ,C (Ed.) (2000). Las mujeres y el poder: representaciones y prácticas de vida. Madrid: al-Mudayna

CORONEL, JM. (1996). La investigación sobre el liderazgo y procesos de cambio en los centros educativos. Huelva: Servicio de Publicaciones de la Universidad, 1996.

CORONEL, J.M., MORENO, E. y PADILLA, Ma.T. (1999). La gestión y el liderazgo como procesos organizativos: contribuciones y retos planteados desde una óptica de género. Revista de Educación.

DÍEZ GUTIÉRREZ, E.J. (2002). Dos visiones de la educación y la calidad. Cuadernos de Pedagogía, 318, noviembre 2002, 84-86.

Díez, E. J.; Valle, E.; Terrón, E. y Centeno, B.: El liderazgo femenino y su ejercicio... Revista Iberoamericana de Educación (ISSN: 1681-5653)

DRAKE, P. y OWEN, P. (Eds). (1998). Gender and Management issues in Education: an international perspective. London: Trentham Books

GAIRÍN, J. Y VILLA, A. (Eds.) (1999): Los equipos directivos de los centros docentes. Análisis de su funcionamiento. Bilbao; Universidad de Deusto.

GAIRIN,J. (1999). Estudios de desarrollo organizativo: de la organización como estructura a la organización que aprende en LORENZO, M. y Otros: Enfoques comparados en organización y dirección de instituciones educativas, Granada, Grupo Editorial Universitario.

GAIRÍN, J. y VILLA, A. (Eds.). (1999). Los equipos directivos de los centros docentes. Análisis de su funcionamiento. Bilbao: Deusto. 
GILLIGAN, C. (1982). In a different voice. Psychological theory and women's development. Cambridge: Harvard University Press.

GIMENO SACRISTAN,J. (1995). La dirección de centros. Análisis de tareas. Madrid: CIDE.

GOLDRING, E. y CHEN, M. (1992). The feminization of principaship in Israel: the trade off between political power and cooperative leadership. Washington and London: Falmer Press.

GRAY, H. (1987). Gender considerations in school management: masculine and femenine leadership styles. School Organization, 7 (3) 297-302.

GRIMWOOD, C. y POPPLESTONE, R. (1993). Women, Management and Care. Londres: McMillan.

GROGAN, M. (1997). Voices of women aspiring to the superintendency. Albany, NY: Suny Press.

HALL, V. (1996). Dancing on the Ceiling: a study of women managers in education London: Paul Chapman.

HELGSEN, S. (1995). The female advantage: Women's ways of leadership Toronto: Doubleday Currency.

HERSEY, S. y BLANCHARD, M. (1993). Management of Organizational Behaviour. Londres: Routledge.

HURTY, K. (1995). Women principals leading with power. New York: SUNY Press.

JACOBS, Jerry. (1999). Gender and the stratification of colleges. The Journal of Higher Education, 70 (2) $161-$

187.

KAUFMANN, A. (1996). Tercer milenio y liderazgo 